\title{
Computed Tomography Findings as Determinants of Local and Systemic Inflammation Biomarkers in Interstitial Lung Diseases: A Retrospective Registry-Based Descriptive Study
}

\author{
David Lang ${ }^{1} \mathbb{D} \cdot$ Kaveh Akbari $^{2} \cdot$ Andreas Horner $^{1,4} \cdot$ Magdalena Hepp $^{1} \cdot$ Bernhard Kaiser $^{1} \cdot$ Herwig Pieringer $^{3}$. \\ Bernd Lamprecht ${ }^{1}$
}

Received: 26 November 2020 / Accepted: 26 February 2021 / Published online: 26 March 2021

(C) The Author(s) 2021

\begin{abstract}
Purpose To evaluate the association of peripheral blood (PBL) and broncho-alveolar lavage (BAL) biomarkers with inflammatory versus fibrotic high-resolution computed tomography (HRCT) findings in interstitial lung disease (ILD) patients.

Methods HRCT findings of 127 consecutive ILD-board patients were semi-quantitatively evaluated: reticulation/honeycombing (RET), traction bronchiectasis (TBR) and emphysema (EMP) were classified as non-inflammatory/fibrotic; consolidations (CON), ground glass opacities (GGO), parenchymal nodules (NDL) and mosaic attenuation (MOS) as active inflammatory. Each HRCT finding was assessed in six distinct lung regions, resulting scores were graded as minimal (0-1 regions involved), medium (2-4) or extensive (5-6). Associations of routinely assessed PBL/BAL biomarkers with these HRCT scores were evaluated using Spearman correlation coefficients and graphical presentation; significance was tested by applying Kruskal-Wallis tests.

Results Blood neutrophil, lymphocyte and eosinophil fraction, neutrophil to lymphocyte ratio (NLR) and BAL lymphocyte fraction consistently showed opposite correlations with inflammatory versus non-inflammatory/fibrotic HRCT finding scores. Blood lymphocyte fraction significantly differed by graded GGO $(p=0.032)$ and CON $(p=0.027)$ extent, eosinophil fraction by TBR $(p=0.006)$ and NLR by CON $(p=0.009)$. C-reactive protein was significantly related to GGO $(p=0.023)$ and CON $(p=0.004)$, BAL lymphocyte fraction to GGO $(p=0.017)$ extent.

Conclusion Blood lymphocyte and eosinophil fraction, NLR, CRP and BAL lymphocyte fraction may aid to differentiate inflammatory from non-inflammatory/fibrotic ILD patterns.

Trial registration This evaluation was based on data from the ILD registry of Kepler University Hospital Linz, as approved by the ethics committee of the Federal State of Upper-Austria (EK Number. I-26-17).
\end{abstract}

Keywords Broncho-alveolar lavage $\cdot$ Peripheral blood $\cdot$ C-reactive protein $\cdot$ Eosinophil $\cdot$ Lymphocyte $\cdot$ Idiopathic pulmonary fibrosis $\cdot$ Chronic hypersensitivity pneumonitis

\section{Introduction}

The anti-fibrotic drugs pirfenidone and nintedanib decelerate lung function decline in idiopathic pulmonary fibrosis (IPF) and progressive fibrosing interstitial lung diseases (PFILD) [1-4], while patients with ILD susceptible to immunomodulatory therapies can experience an improvement in both radiological imaging and pulmonary function

David Lang

david.lang@kepleruniklinikum.at

Extended author information available on the last page of the article tests [5-7]. Still, also ILD with an "inflammatory" origin like systemic sclerosis associated ILD (SSCILD) or chronic hypersensitivity pneumonitis (CHP) frequently present with a PFILD phenotype [8, 9]. A combination of prednisone, azathioprine and $\mathrm{N}$-acetylcysteine led to adverse outcomes in IPF patients [10, 11], while in SSCILD placebo-controlled trials have provided evidence on the efficacy of immunosuppressive as well as anti-fibrotic therapies $[6,12,13]$. In various fibrotic ILD apart from IPF it is still unclear, whether an anti-fibrotic, an immunosuppressive or a combined approach is most beneficial. 
In current clinical practice in ILD, most biomarker information is derived from high-resolution computed tomography (HRCT) imaging. A radiological pattern of usual interstitial pneumonia (UIP) irrespective of the underlying etiology bears a poorer prognosis than possible UIP or nonspecific interstitial pneumonia (NSIP) [14-20]. Under certain conditions, an UIP-pattern can be diagnostic for IPF and lead to the initiation of anti-fibrotic therapy without the need of lung biopsy [19, 20]. In most ILD cases, however, HRCT patterns are not uniform but rather involve several coexisting abnormalities for example reticulation (RET), ground glass opacities (GGO) and traction bronchiectasis (TBR) in fibrotic NSIP. The relative distribution and extent of such radiological findings depends on the underlying pathogenetic processes, the course and duration of disease [15].

Hypothetically, peripheral blood (PBL) and bronchoalveolar lavage (BAL) biomarkers could help to differentiate ILD cases with an inflammatory from those with a predominantly fibrotic phenotype. Knowledge on their interaction with HRCT findings could aid the development of biomarkers guiding ILD therapy in the future.

\section{Methods}

Based on a retrospective ILD registry cohort, we have evaluated routinely assessed biomarkers from PBL and BAL fluid for their association with a set of visually semiquantified HRCT finding scores.

This study was performed according to the Strengthening the Reporting of Observational Studies in Epidemiology (STROBE) guidelines for reporting observational studies [21]. Patient data used for this analysis were retrieved from the ILD registry of Kepler University Hospital Linz, Austria. The registry as well as the present evaluation have been conducted in concordance with the Declaration of Helsinki and were approved and reassessed on a yearly basis by the ethics committee of the Federal State of Upper Austria (Study number I-26-17). All patients enrolled were subsequently discussed by the monthly local ILD-board after they had undergone a standardized ILD evaluation program including a structured assessment of patient history and physical examination, HRCT imaging, pulmonary function tests and laboratory analyses with standard autoimmune serologies [20, 22]. Patients in whom ILD board discussion resulted in no ILD diagnosis were excluded from this study.

HRCT images were acquired according to protocols suggested by the relevant guidelines [19, 20, 23]. If clinically feasible, prone imaging was preferred to differ opacities in dependent lung areas from true interstitial lung abnormalities [24].
Blood samples were analyzed using a Sysmex $®$ XN-3000 hematology analyzer (Sysmex Europe GmbH, Norderstedt, Germany) for blood cell counts and a Cobas ${ }^{\circledR} 8000$ modular analyzer (Roche Diagnostics International AG, Rotkreuz, Switzerland) for C-reactive protein (CRP) and lactate dehydrogenase (LDH).

Broncho-alveolar lavage was performed according to the relevant guidelines $[25,26]$, when clinically indicated by the treating physician or by the ILD-board. A total of $100 \mathrm{~mL}$ of $0.9 \%$ saline was instilled and retrieved in aliquots of $20 \mathrm{~mL}$ via flexible bronchoscopy under sedoanalgesia. The BAL location was a segmental bronchus of either one of the upper lobes including the lingula or the middle lobe at the discretion of the conducting physician according to the location of most active or extensive disease in HRCT. BAL samples were prepared using $100 \mu \mathrm{L}$ of BAL fluid on a Tharmac ${ }^{\circledR}$ Cellspin I cytocentrifuge (Tharmac GmbH, Wiesbaden, Germany) at 700 rounds per minute for $5 \mathrm{~min}$ and Wright Giemsa staining. Cell counting was performed manually under 400-fold magnification, cell fractions were given as $\%$ of the total cell count, excluding epithelial cells or erythrocytes. Further lymphocyte subset analyses were performed in BAL samples with a lymphocyte fraction of $\geq 15 \%$.

To allow for statistical analyses of HRCT scans, we have previously devised a semi-quantitative scoring system based on four elementary lesion types: parenchymal nodules (NDL), reticular abnormalities (interlobular septal and intralobular interstitial thickening and honeycombingRET), increased lung attenuation (consolidations (CON), ground glass opacities (GGO)) and reduced lung attenuation (emphysema-EMP) findings. Besides, the extent of mosaic attenuation (mosaic perfusion, air-trapping-MOS) and traction bronchi(-ol)ectasis (TBR) were assessed. [23, $27,28]$. For quantification, both lungs were separated in an upper-, middle- and lower-lung area as defined by thirds of the largest cranio-caudal diameter in the sagittal reconstructions, leading to six distinct lung areas. The individual extent of each quantified HRCT pattern (RET, TBR, EMP, CON, GGO, NDL, MOS) was calculated as the sum of all involved defined lung areas (0-6). The described HRCT scoring process was accomplished during the respective ILD-board session by a specialized ILD radiologist in a non-blinded fashion.

To evaluate the associations between PBL and BAL inflammation biomarkers and the quantified imaging features, correlation coefficients were calculated for each HRCT finding score and each PBL and BAL biomarker. Direction, strength, and significance of these correlations were depicted in color-coded tables for visual analysis. To test for clinically relevant significance of these associations, groups with no or minimal (0-1), medium (2-4) and extensive HRCT involvement (5-6) were compared using the Kruskal-Wallis test. All statistical analyses were performed 
using R (R: A Language and Environment for Statistical Computing; Version 3.6.0; https://www.R-project.org). For all tests performed, a $p$-value $<0.05$ was regarded statistically significant.

\section{Results}

We evaluated 127 ILD patients consecutively discussed by the multidisciplinary ILD-board of Kepler University Hospital Linz, Austria between February 2017, and September 2018. Clinical and radiological patient characteristics are shown in Tables 1 and 2 . The fraction of patients with "other ILD" $(n=23 ; 18 \%)$ included nine cases of organizing pneumonia (OP), six patients with sarcoidosis, three with respiratory-bronchiolitis-ILD, two with drug-associated pneumonitis and one patient each with pulmonary Langerhans-cell histiocytosis, pleuro-parenchymal fibroelastosis and lymphangioleiomyomatosis. Eleven patients (9\%) were considered "unclassified ILD", either due to patients not willing to undergo further necessary diagnostic steps like lung biopsy or to situations, where further work-up was deemed inappropriate due to age or major comorbidities. Eleven patients (9\%; ten with NSIP, one with unclassifiable ILD) were or had already been diagnosed with autoimmune disorders considered causally related to ILD (rheumatoid arthritis in four patients, autoimmune-hepatitis in two patients, Sjögren's syndrome in two patients and pauci-immune glomerulonephritis, granulomatosis with polyangiitis and SHARPsyndrome in one patient each).

HRCT, PBL and BAL characteristics according to ILDboard diagnoses are shown in Supplementary Table 1.

Correlations of PBL and BAL biomarkers with HRCT finding scores are shown in Fig. 1. Blood neutrophil, lymphocyte and eosinophil fraction, neutrophil to lymphocyte ratio (NLR) and BAL lymphocyte fraction showed consistently opposite correlations for inflammatory versus noninflammatory/fibrotic HRCT finding scores. Significant correlations were seen for PBL lymphocyte fraction and GGO $(r=-0.27, p<0.01)$, PBL NLR and GGO $(r=0.23$, $p<0.05)$ and for PBL eosinophil fraction and RET $(r=0.2$, $p<0.05)$ as well as TBR $(r=0.25, p<0.01)$. Concerning

Table 1 Patient characteristics

\begin{tabular}{|c|c|c|c|}
\hline Patient characteristics & & Comorbidities & $n(\%)$ \\
\hline Mean age (years, SD) & $65(14)$ & None & $17(13)$ \\
\hline Age range (years) & $18-91$ & Pulmonary & \\
\hline Male sex $(n, \%)$ & $82(65)$ & Chronic obstructive pulmonary disease & $15(12)$ \\
\hline Reported onset of respiratory symptoms (years, SD) & $4.1(5.8)$ & Asthma & $8(6)$ \\
\hline \multirow[t]{3}{*}{ Family history of ILD $(n, \%)$} & $8(6)$ & History of tuberculosis & $7(6)$ \\
\hline & & Cardiovascular & \\
\hline & & Arterial hypertension & $46(36)$ \\
\hline \multirow[t]{2}{*}{ ILD-board diagnosis } & $n(\%)$ & Coronary artery disease & $22(17)$ \\
\hline & & Atrial fibrillation & $19(15)$ \\
\hline Interstitial pneumonia with autoimmune features & $26(20)$ & Chronic kidney disease & $8(6)$ \\
\hline Idiopathic pulmonary fibrosis & $23(18)$ & Stroke/cerebrovascular disease & $6(5)$ \\
\hline Idiopathic non-specific interstitial pneumonia & $16(13)$ & Peripheral artery disease & $5(4)$ \\
\hline Associated ILD & $11(9)$ & Endocrine & \\
\hline Unclassified ILD & $11(9)$ & Diabetes mellitus & $19(15)$ \\
\hline \multirow[t]{3}{*}{ Other ILD } & $23(18)$ & Thyroid disorder & $13(10)$ \\
\hline & & Hyperlipidemia & $10(8)$ \\
\hline & & Osteoporosis & $5(4)$ \\
\hline Smoking history & $n(\%)$ & Gastrointestinal & \\
\hline Mean pack years (mean, SD) & $19.4(25.2)$ & Gastroesophageal reflux disease & $17(13)$ \\
\hline Never smoker & $52(41)$ & Viral hepatitis & $7(6)$ \\
\hline Former smoker & $50(39)$ & Autoimmune & \\
\hline Current smoker & $17(13)$ & Related to ILD* & $11(9)$ \\
\hline \multirow[t]{2}{*}{ Exclusively passive smoker } & $6(5)$ & Psoriasis & $7(6)$ \\
\hline & & Malignancy (solid and haematological) & $12(9)$ \\
\hline
\end{tabular}

Data are given as $n(\%)$ unless otherwise specified. Only comorbidities present in five or more patients are listed

*Specified in the results section

$S D$ Standard deviation, ILD Interstitial lung disease 
Table 2 Peripheral blood, broncho-alveolar lavage, and HRCT characteristics

\begin{tabular}{|c|c|c|}
\hline Peripheral blood biomarkers & $n(\%)$ & Mean (SD) \\
\hline Leukocyte count (G/L) & $122(96)$ & $8.7(3.4)$ \\
\hline Neutrophil fraction (\%) & $121(95)$ & $70.9(10.9)$ \\
\hline Lymphocyte fraction (\%) & & $20.5(8.7)$ \\
\hline Neutrophil to lymphocyte ratio & & $5(4.9)$ \\
\hline Eosinophil fraction (\%) & & $0.2(0.3)$ \\
\hline C-reactive protein $(\mathrm{mg} / \mathrm{dL})$ & $123(97)$ & $1.6(2.6)$ \\
\hline Lactate dehydrogenase (U/L) & $113(89)$ & $247(78.2)$ \\
\hline BAL biomarkers & $n(\%)$ & mean $(\mathrm{SD})$ \\
\hline Macrophage fraction (\%) & $66(52)$ & $51.9(29.9)$ \\
\hline Neutrophil fraction (\%) & & $18.5(23.1)$ \\
\hline Lymphocyte fraction (\%) & & $18.5(21.6)$ \\
\hline Eosinophil fraction (\%) & & $3.6(7.9)$ \\
\hline $\mathrm{CD} 4+/ \mathrm{CD} 8+$ ratio & $20(16)$ & $2.3(1.9)$ \\
\hline HRCT finding scores & $n(\%)$ & Median (range) \\
\hline Parenchymal nodules & $40(31)$ & $0(0-6)$ \\
\hline Reticulation/honeycombing & $106(83)$ & $4(0-6)$ \\
\hline Honeycombing & $22(17)$ & $0(0-6)$ \\
\hline Ground glass opacities & 49 (39) & $0(0-6)$ \\
\hline Consolidations & $44(35)$ & $0(0-6)$ \\
\hline Emphysema & $23(18)$ & $0(0-6)$ \\
\hline Traction bronchiectasis & $100(79)$ & $2(0-6)$ \\
\hline Mosaic attenuation & $32(25)$ & $0(0-6)$ \\
\hline
\end{tabular}

Values are given as $n(\%)$ and mean (SD) or median (range) as specified

$S D$ Standard deviation, IQR Interquartile range, BAL Broncho-alveolar lavage, $C D$ Cluster of differentiation, HRCT High-resolution computed tomography

CRP, GGO $(r=0.23, p<0.05)$ and $\mathrm{CON}(r=0.21, p<0.05)$ showed significant interactions, while LDH was significantly correlated to RET $(\mathrm{r}=0.29, p<0.01)$, TBR $(r=0.43$, $\mathrm{p}<0.01)$, GGO $(r=0.24, p<0.05)$ and MOS $(r=0.3$, $p<0.01)$. Among BAL biomarkers, only lymphocyte fraction was significantly correlated with HRCT finding scores, namely with GGO $(r=0.34, p<0.01)$ and CON $(r=0.35$, $p<0.01$ ).

As shown in Figs. 2 and 3 as well as in Supplementary Tables 2 and 3, the categorized scores for the extent of HRCT findings were used to test for clinically meaningful significance of the associations with each PBL or BAL biomarker variable. Blood lymphocyte fraction significantly differed by graded GGO ( $p=0.032$, Fig. 2a) and CON $(p=0.027$, Fig. $2 \mathrm{~b})$, blood NLR by CON $(p=0.009$, Fig. $2 \mathrm{c})$ and blood eosinophil fraction by TBR ( $p=0.006$, Fig. $2 \mathrm{~d})$ extent. CRP was significantly related to GGO $(p=0.023$, Fig. 2e) and CON ( $p=0.004$, Fig. 2f), while LDH showed significant associations with RET ( $p=0.01$, Fig. 2 g), TBR $(p<0.001$, Fig. 2 h), GGO ( $p=0.049$, Fig. $2 \mathrm{i})$ and MOS $(p=0,027$, Fig. 2j).
Concerning BAL biomarkers, the only significant interaction was shown for BAL lymphocyte fraction and GGO $(p=0.017$, Fig. 3). Analyses on BAL cluster of differentiation (CD) $4+/ \mathrm{CD} 8+$ ratio were only available in a minority of patients and revealed close association with the extent of parenchymal nodules (Supplementary Figure 1 and Supplementary Table 4).

\section{Discussion}

In summary, our analyses indicate that PBL lymphocyte and eosinophil fraction, neutrophil to lymphocyte ratio (NLR), CRP as well as BAL lymphocyte fraction may have clinically relevant implications in differing HRCT abnormalities indicating either active inflammation (ground glass opacities, consolidations, parenchymal nodules, mosaic attenuation) or non-inflammatory/fibrotic processes (reticulation/ honeycombing, traction bronchiectasis, emphysema).

Blood lymphocyte count is of known prognostic relevance in systemic disorders like SSC or malignancy 


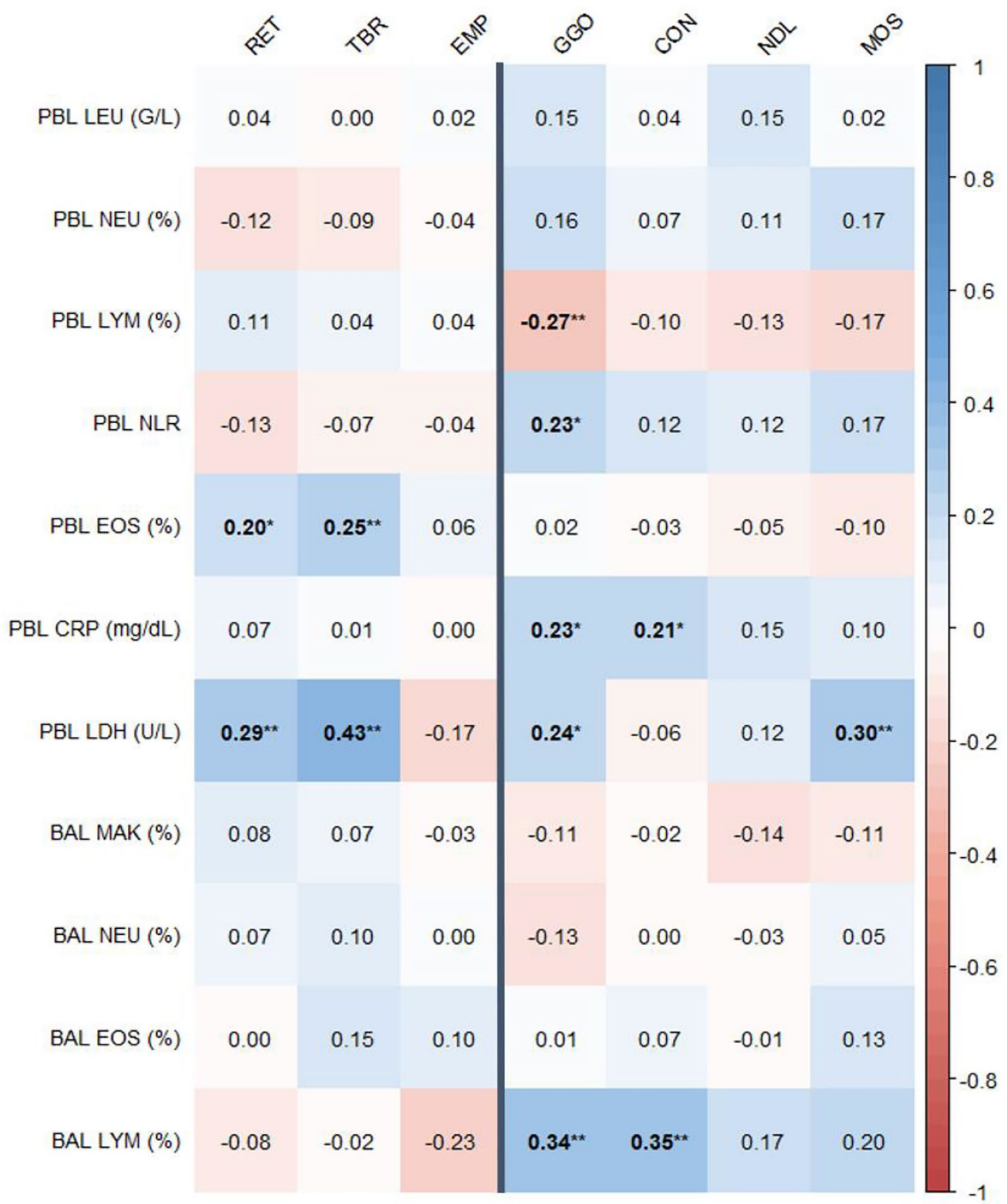

Fig. 1 Correlation matrix of peripheral blood and broncho-alveolar lavage biomarkers with HRCT finding scores. Values are for Spearman correlation coefficients; colors indicate strength and direction of correlations as shown by the scale on the right side. Bold numbers are for significant correlations, $* p<0.05, * * p<0.01$. The line between the EMP and GGO category visually separates non-inflammatory/ fibrotic from inflammatory HRCT findings. $P B L$ Peripheral blood, $B A L$ Broncho-alveolar lavage, HRCT High-resolution computed tomography, RET Reticulation/honeycombing, TBR Traction bronchiectasis, EMP Emphysema, GGO Ground glass opacities, CON Consolidations, NDL Parenchymal nodules, MOS Mosaic attenuation, $L E U$ Leukocyte count, NEU Neutrophil fraction, $L Y M$ Lymphocyte fraction, $N L R$ Neutrophil to lymphocyte ratio, EOS Eosinophil fraction, $C R P$ C-reactive protein, $L D H$ Lactate dehydrogenase, $M A K$ Macrophage fraction 


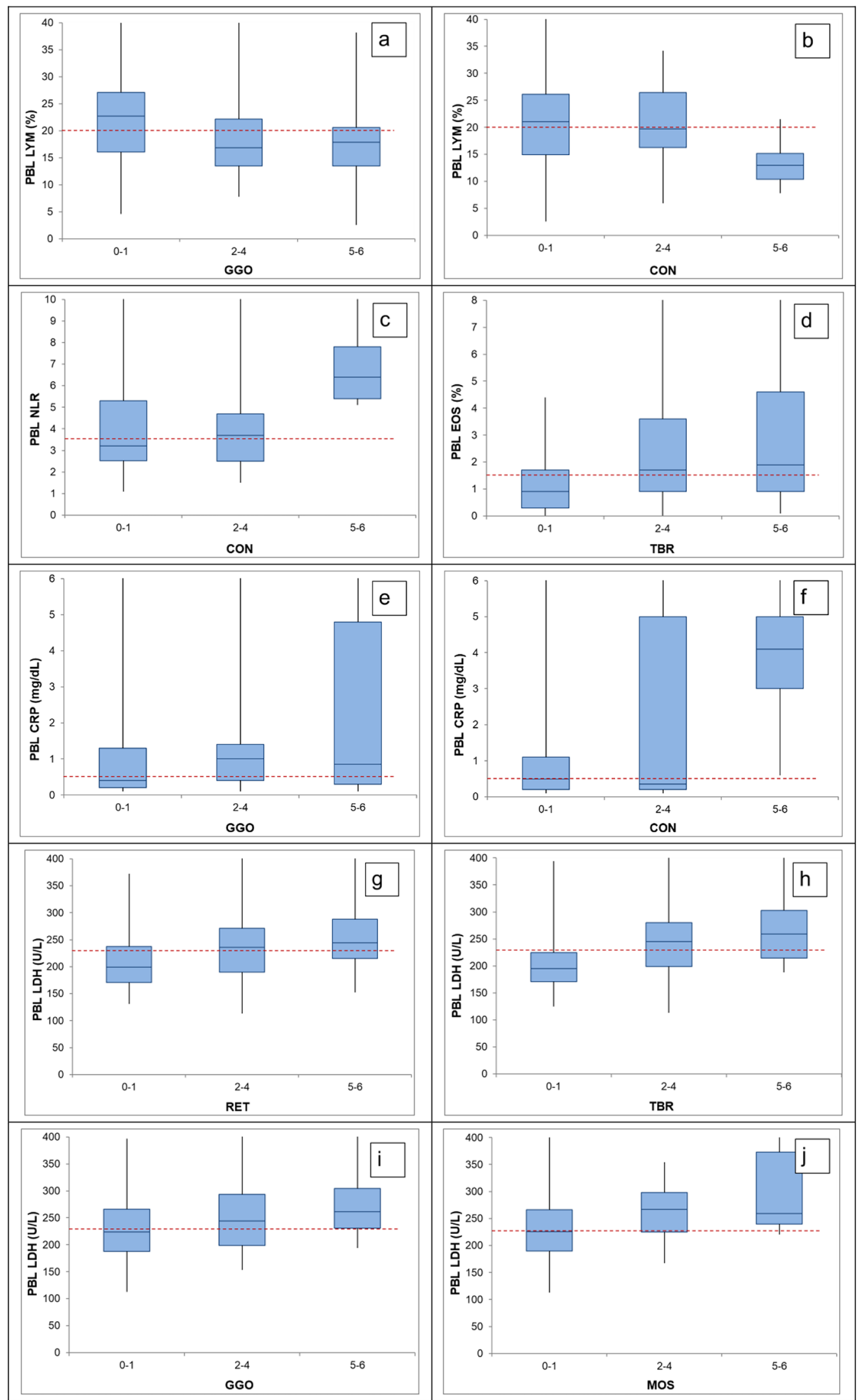


४Fig. 2 Boxplot diagrams of significant findings for PBL biomarkers according to HRCT score categories. Boxplot diagrams depict the median (line), the upper and lower quartile (boxes) and the $95 \%$ confidence intervals (whiskers). The red dashed line represents the median of all values. PBL Peripheral blood, $L Y M$ Lymphocyte fraction, GGO Ground glass opacities, CON Consolidations, NLR Neutrophil to lymphocyte ratio, EOS Eosinophil fraction, TBR Traction bronchiectasis, $C R P$ C-reactive protein, $L D H$ Lactate dehydrogenase, RET Reticulation/honeycombing, MOS Mosaic attenuation, HRCT High-resolution computed tomography

[29-31]. Our finding that lymphocyte fraction was consistently negatively correlated with the extent of HRCT abnormalities considered "inflammatory" suggests that similar mechanisms may be present in ILD. In BAL, however, lymphocyte fraction showed the opposite behavior with distinctly higher values in the presence of GGO and CON, but also NOD and MOS. Lymphocytosis in BAL is a common finding in inflammatory ILD presenting with patterns of NSIP or OP [5, 32, 33], and especially in ILD associated with formation of granulomas, such as sarcoidosis or hypersensitivity pneumonitis [25]. Conversely, BAL lymphocyte counts are reportedly lower with increasing fibrosis [7, 34].

Similar to our observation of PBL eosinophil fraction being positively correlated with RET and TBR, blood eosinophilia has been reported to be associated with disease severity and presence of ILD in SSC [35]. Elevated eosinophil counts in BAL fluid have repeatedly been described in IPF patients as well as in fibrotic rather than in cellular NSIP [7, 32, 34]. Furthermore, it has been suggested that higher BAL eosinophil counts in IPF patients are associated with an increased risk of acute exacerbations and inferior survival rates [36, 37]. In our patient cohort, such findings for BAL eosinophil fraction could not be shown, possibly due to the low number of patients presenting with significant BAL eosinophilia. The role of eosinophils in the pathogenesis of ILD has not been comprehensively understood yet: cytokines involved in eosinophil activation like Interleukin(IL)-4, IL-5, IL-13 or IL-33 also play a major role in the pathophysiology of IPF and SSCILD [10, 35, 38, 39]. However, it is still unknown, whether an increased eosinophil count in blood or BAL fluid has fundamental pathogenetic implications or rather represents a bystander phenomenon of the molecular processes underlying pulmonary fibrosis. Our findings and the limited existing evidence suggest that an increased eosinophil count may more likely be an indicator of ILD severity and extent than a causative factor.

The close relationship observed between CRP and GGO/ CON resembles reports of elevated CRP in ILD patients presenting with patterns like NSIP or OP [5, 7, 15]. In ILD associated with autoimmune disorders, alterations to systemic inflammatory parameters like CRP have been frequently reported. They also pose a risk factor for the development of pulmonary involvement in such conditions [30, 40-42]. Contrary to CRP, we found that lactate dehydrogenase (LDH) was significantly positively correlated with multiple both non-inflammatory/fibrotic and inflammatory HRCT finding scores. Elevation in LDH has been reported in IPF, where it may be associated with functional impairment and may have prognostic properties [43]. Our findings however suggest that LDH could rather be a biomarker reflecting general disease extent and severity than the underlying pathogenetic processes.

Several limitations to our reported study need to be addressed: next to its retrospective, single center approach, the sample size was limited. The reported collective represents a heterogeneous group of several different ILD entities, also including a minority of patients without signs of reticulation or honeycombing (17\%). The study collective was derived from patients subsequently discussed by the local ILD-board, which could have increased the likelihood of inclusion of rather complex ILD cases, while typical ILD like for example sarcoidosis may be underrepresented. However, our reported evaluation explicitly did not focus on distinct ILD diagnoses, but on HRCT imaging findings and their association with biomarkers of systemic and local inflammation. Radiological assessment was not accomplished in a blinded fashion but in the presence of the ILD-board, which reflects the multidisciplinary approach to ILD. Our reported scoring system has not been validated in a larger patient cohort but is simple to perform and does not require additional tools like special software. It was not our aim to create a comprehensive HRCT quantification and classification tool but to allow semiquantitative statistical analyses of HRCT scans beyond only "present or absent". It is obvious that PBL cell counts and biomarkers like CRP or LDH can be substantially altered by infections, neoplastic or hematological conditions. Also, BAL differential cell counts can be influenced by presence of infection, smoking status or age $[26,44]$. Additionally, BAL was only performed in approximately half of the patient collective, for it had either been done previously or it was deemed clinically unnecessary. For BAL analyses, this could have led to a selection of patients with uncommon presentation in HRCT or with rather acute than chronic ILD, as suggested by the comparably high mean lymphocyte and neutrophil counts reported. Concerning statistical methods, we acknowledge, that numerous associations have been evaluated for statistical significance, which brings up the issue of multiple testing. Concerning this matter, it was not our 
Fig. 3 Boxplot diagram of BAL lymphocyte fraction according to GGO score categories. Boxplot diagrams depict the median (line), the upper and lower quartile (boxes) and the $95 \%$ confidence intervals (whiskers). The red dashed line represents the median of all values. $B A L$ Broncho-alveolar lavage, $L Y M$ Lymphocyte fraction, $G G O$ ground glass opacities

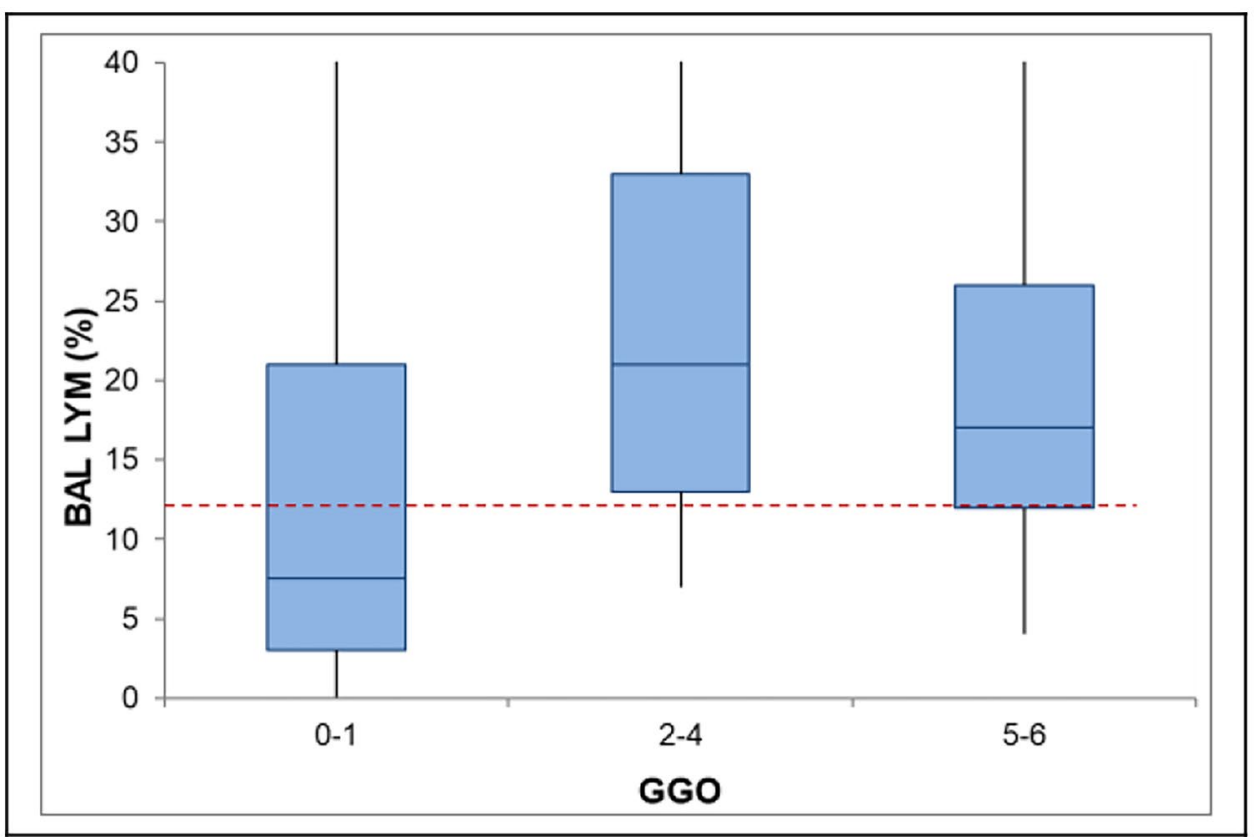

aim to test for significance of certain associations, but rather to apply an experimental, hypothesis-building approach aiming to extract clinically relevant biomarkers from a large, diverse dataset. Seeking to overcome these methodological challenges, we primarily used descriptive statistical evaluation like Spearman correlation coefficients and graphical presentation. We chose clinically meaningful quantification categories (no or little, medium, or abundant involvement) for HRCT findings and used the Kruskal-Wallis test for significance testing due to its robustness against outliers.

\section{Conclusion}

We conclude that blood lymphocyte and eosinophil fraction, NLR, CRP and BAL lymphocyte fraction may help to differentiate between non-inflammatory/fibrotic and active inflammatory ILD phenotypes. Especially in ILD with multiple coexisting HRCT abnormalities, these biomarkers could aid the decision whether to primarily initiate anti-inflammatory or antifibrotic treatment. Subsequent prospective and largerscale trials are warranted to further evaluate the implications of these biomarkers on response to either therapeutic approach.

Supplementary Information The online version contains supplementary material available at https://doi.org/10.1007/s00408-021-00434-w.

Author Contributions All authors have approved the paper, are able to verify the validity of the results reported and meet the criteria for authorship as established by the International Committee of Medical Journal Editors. Conceptualization, DL, KA, HP and BL;
Methodology, DL, AH, BK, HP and BL; Software, BK; Validation, DL, KA, AH, MH, and BK; Formal analysis, DL and BK; Investigation, DL, KA, AH, MH and BL; Resources, DL, KA and BL; Data Curation, DL, KA and BK; Writing-Original Draft Preparation, DL, $\mathrm{KA}, \mathrm{AH}$ and $\mathrm{MH}$; Writing-Review \& Editing, DL, KA, AH, MH, BK, HP and BL; Visualization, DL, AH, BK, HP and BL; Supervision, DL and BL; Project Administration, DL and BL; Funding Acquisition, not applicable.

Funding Open access funding provided by Johannes Kepler University Linz. No external funding was used in conducting the study or in writing this manuscript.

Data Availability According to the terms imposed by the ethics committees, the full dataset analyzed during the current study cannot be made publicly available, as it contains possibly identifiable patient data. Upon reasonable request to the corresponding author and if approved as an amendment by the responsible local ethics committee, selected anonymized data can however be shared.

\section{Compliance with Ethical Standards}

Conflicts of interest The authors declare that they have no conflict of interest.

Ethical Approval This study was conducted in accordance with the Declaration of Helsinki and approved by the ethics committee of the federal state of Upper-Austria (EK Nr. I-26-17). It was conducted in an entirely retrospective fashion, without an experimental approach or additional patient contact. Only patient data assessed in clinical routine were analyzed. Data were collected in an anonymized fashion and securely electronically stored in a way that only the authors had access to the data. No identifiable patient data has been or will ever be published by the authors. 
Consent to Participate According to the ethic committee approval, no patient consent was necessary for participation in this study.

Open Access This article is licensed under a Creative Commons Attribution 4.0 International License, which permits use, sharing, adaptation, distribution and reproduction in any medium or format, as long as you give appropriate credit to the original author(s) and the source, provide a link to the Creative Commons licence, and indicate if changes were made. The images or other third party material in this article are included in the article's Creative Commons licence, unless indicated otherwise in a credit line to the material. If material is not included in the article's Creative Commons licence and your intended use is not permitted by statutory regulation or exceeds the permitted use, you will need to obtain permission directly from the copyright holder. To view a copy of this licence, visit http://creativecommons.org/licenses/by/4.0/.

\section{References}

1. Richeldi L, Cottin V, du Bois RM et al (2016) Nintedanib in patients with idiopathic pulmonary fibrosis: combined evidence from the TOMORROW and INPULSIS $®$ trials. Respir Med 113:74-79. https://doi.org/10.1016/j.rmed.2016.02.001

2. Noble PW, Albera C, Bradford WZ et al (2016) Pirfenidone for idiopathic pulmonary fibrosis: analysis of pooled data from three multinational phase 3 trials. Eur Respir J 47:243-253. https://doi. org/10.1183/13993003.00026-2015

3. Maher TM, Corte TJ, Fischer A et al (2019) Pirfenidone in patients with unclassifiable progressive fibrosing interstitial lung disease: a double-blind, randomised, placebo-controlled, phase 2 trial. Lancet Respir Med. https://doi.org/10.1016/S2213-2600(19) 30341-8

4. Flaherty KR, Wells AU, Cottin V et al (2019) Nintedanib in progressive fibrosing interstitial lung diseases. N Engl J Med 381:1718-1727. https://doi.org/10.1056/NEJMoa1908681

5. Ito Y, Arita M, Kumagai S et al (2017) Serological and morphological prognostic factors in patients with interstitial pneumonia with autoimmune features. BMC Pulm Med 17:111. https://doi. org/10.1186/s12890-017-0453-Z

6. Tashkin DP, Roth MD, Clements PJ et al (2016) Mycophenolate mofetil versus oral cyclophosphamide in scleroderma-related interstitial lung disease (SLS II): a randomised controlled, double-blind, parallel group trial. Lancet Respir Med 4:708-719. https://doi.org/10.1016/S2213-2600(16)30152-7

7. Park IN, Jegal Y, Kim DS et al (2009) Clinical course and lung function change of idiopathic nonspecific interstitial pneumonia. Eur Respir J 33:68-76. https://doi.org/10.1183/09031936. 00158507

8. Kolb M, Vašáková M (2019) The natural history of progressive fibrosing interstitial lung diseases. Respir Res 20:57. https://doi. org/10.1186/s12931-019-1022-1

9. Wijsenbeek M, Cottin V (2020) Spectrum of fibrotic lung diseases. N Engl J Med 383:958-968. https://doi.org/10.1056/ NEJMra2005230

10. Heukels P, Moor CC, von der Thüsen JH et al (2019) Inflammation and immunity in IPF pathogenesis and treatment. Respir Med 147:79-91. https://doi.org/10.1016/j.rmed.2018.12.015

11. Idiopathic Pulmonary Fibrosis Clinical Research Network, Raghu G, Anstrom KJ et al (2012) Prednisone, azathioprine, and $N$-acetylcysteine for pulmonary fibrosis. N Engl J Med 366:1968-77. https://doi.org/10.1056/NEJMoa1113354

12. Khanna D, Denton CP, Lin CJF et al (2018) Safety and efficacy of subcutaneous tocilizumab in systemic sclerosis: results from the open-label period of a phase II randomised controlled trial
(faSScinate). Ann Rheum Dis 77:212-220. https://doi.org/10. 1136/annrheumdis-2017-211682

13. Distler O, Highland KB, Gahlemann M et al (2019) Nintedanib for systemic sclerosis-associated interstitial lung disease. $\mathrm{N}$ Engl J Med 380:2518-2528. https://doi.org/10.1056/NEJMo a1903076

14. Walsh SLF, Devaraj A, Enghelmayer JI et al (2018) Role of imaging in progressive-fibrosing interstitial lung diseases. Eur Respir Rev 27:180073. https://doi.org/10.1183/16000617. 0073-2018

15. Jeny F, Brillet P-Y, Kim Y-W et al (2019) The place of highresolution computed tomography imaging in the investigation of interstitial lung disease. Expert Rev Respir Med 13:79-94. https:// doi.org/10.1080/17476348.2019.1556639

16. Salisbury ML, Tolle LB, Xia M et al (2017) Possible UIP pattern on high-resolution computed tomography is associated with better survival than definite UIP in IPF patients. Respir Med 131:229 235. https://doi.org/10.1016/j.rmed.2017.08.025

17. Flaherty KR (2003) Radiological versus histological diagnosis in UIP and NSIP: survival implications. Thorax 58:143-148. https:// doi.org/10.1136/thorax.58.2.143

18. Yunt ZX, Chung JH, Hobbs S et al (2017) High resolution computed tomography pattern of usual interstitial pneumonia in rheumatoid arthritis-associated interstitial lung disease: relationship to survival. Respir Med 126:100-104. https://doi.org/10.1016/j. rmed.2017.03.027

19. Lynch DA, Sverzellati N, Travis WD et al (2018) Diagnostic criteria for idiopathic pulmonary fibrosis: a Fleischner Society White Paper. Lancet Respir Med 6:138-153. https://doi.org/10. 1016/S2213-2600(17)30433-2

20. Raghu G, Remy-Jardin M, Myers JL et al (2018) Diagnosis of idiopathic pulmonary fibrosis. An official ATS/ERS/JRS/ALAT clinical practice guideline. Am J Respir Crit Care Med 198:e44e68. https://doi.org/10.1164/rccm.201807-1255ST

21. von Elm E, Altman DG, Egger M et al (2007) The Strengthening the Reporting of Observational Studies in Epidemiology (STROBE) statement: guidelines for reporting observational studies. Lancet 370:1453-1457. https://doi.org/10.1016/S01406736(07)61602-X

22. Fischer A, Antoniou KM, Brown KK et al (2015) An official European Respiratory Society/American Thoracic Society research statement: interstitial pneumonia with autoimmune features. Eur Respir J 46:976-987. https://doi.org/10.1183/13993003. 00150-2015

23. Gruden JF, Naidich DP, Machnicki SC et al (2019) An algorithmic approach to the interpretation of diffuse lung disease on chest CT imaging. Chest. https://doi.org/10.1016/j.chest.2019. 10.017

24. Kim M, Lee SM, Song J-W et al (2017) Added value of prone CT in the assessment of honeycombing and classification of usual interstitial pneumonia pattern. Eur J Radiol 91:66-70. https://doi. org/10.1016/j.ejrad.2017.03.018

25. Meyer KC (2014) The clinical utility of bronchoalveolar lavage in interstitial lung disease-is it really useful? Expert Rev Respir Med 8:133-135. https://doi.org/10.1586/17476348.2014.879827

26. Meyer KC, Raghu G, Baughman RP et al (2012) An official American Thoracic Society clinical practice guideline: the clinical utility of bronchoalveolar lavage cellular analysis in interstitial lung disease. Am J Respir Crit Care Med 185:1004-1014. https://doi. org/10.1164/rccm.201202-0320ST

27. Lang D, Akbari K, Lamprecht B, Fellner F (2018) Initial patient characteristics and standardized quantification of radiological findings in nonspecific interstitial pneumonia. Pneumologie 72:S48-S48. https://doi.org/10.1055/s-0037-1619245

28. Lang D, Akbari K, Horner A et al (2020) Standardized computed tomography findings as determinants of local and systemic 
inflammation in interstitial lung disease patients. In: B42 ILD Epidemiol I [Internet]. American Thoracic Society, pp A3392A3392. https://www.atsjournals.org/doi/10.1164/ajrccm-confe rence.2020.201.1_MeetingAbstracts.A3392

29. Gambichler T, Tigges C, Burkert B et al (2010) Absolute count of $\mathrm{T}$ and B lymphocyte subsets is decreased in systemic sclerosis. Eur J Med Res 15:44. https://doi.org/10.1186/2047-783X-15-1-44

30. Bonhomme O, André B, Gester F et al (2019) Biomarkers in systemic sclerosis-associated interstitial lung disease: review of the literature. Rheumatology 58:1534-1546. https://doi.org/10.1093/ rheumatology/kez230

31. Huemer F, Lang D, Westphal T et al (2019) Baseline absolute lymphocyte count and ECOG performance score are associated with survival in advanced non-small cell lung cancer undergoing PD-1/PD-L1 blockade. J Clin Med 8:1014. https://doi.org/10. 3390/jcm8071014

32. Welker L (2004) Predictive value of BAL cell differentials in the diagnosis of interstitial lung diseases. Eur Respir J 24:1000-1006. https://doi.org/10.1183/09031936.04.00101303

33. Costabel U, Bonella F, Ohshimo S, Guzman J (2010) Diagnostic modalities in sarcoidosis: BAL, EBUS, and PET. Semin Respir Crit Care Med 31:404-408. https://doi.org/10.1055/s-0030-1262207

34. Veeraraghavan S, Latsi PI, Wells AU et al (2003) BAL findings in idiopathic nonspecific interstitial pneumonia and usual interstitial pneumonia. Eur Respir J 22:239-244. https://doi.org/10.1183/ 09031936.03.00105202

35. Ando K, Nakashita T, Kaneko N et al (2016) Associations between peripheral blood eosinophil counts in patients with systemic sclerosis and disease severity. Springerplus 5:1401. https:// doi.org/10.1186/s40064-016-3106-4

36. Kakugawa T, Sakamoto N, Sato $\mathrm{S}$ et al (2016) Risk factors for an acute exacerbation of idiopathic pulmonary fibrosis. Respir Res 17:79. https://doi.org/10.1186/s12931-016-0400-1

37. Boomars KA, Wagenaar SS, Mulder PG et al (1995) Relationship between cells obtained by bronchoalveolar lavage and survival in idiopathic pulmonary fibrosis. Thorax 50:1087-1092. https://doi. org/10.1136/thx.50.10.1087
38. Vettori S, Cuomo G, Iudici M et al (2014) Early systemic sclerosis: serum profiling of factors involved in endothelial, T-cell, and fibroblast interplay is marked by elevated interleukin-33 levels. J Clin Immunol 34:663-668. https://doi.org/10.1007/ s10875-014-0037-0

39. Davoine F, Lacy P (2014) Eosinophil cytokines, chemokines, and growth factors: emerging roles in immunity. Front Immunol. https://doi.org/10.3389/fimmu.2014.00570

40. Zhang L, Wu G, Gao D et al (2016) Factors associated with interstitial lung disease in patients with polymyositis and dermatomyositis: a systematic review and meta-analysis. PLoS ONE 11:e0155381. https://doi.org/10.1371/journal.pone.0155381

41. Gao H, Zhang X-W, He J et al (2018) Prevalence, risk factors, and prognosis of interstitial lung disease in a large cohort of Chinese primary Sjögren syndrome patients. Medicine (Baltimore) 97:e11003. https://doi.org/10.1097/MD.0000000000011003

42. Liu X, Mayes MD, Pedroza C et al (2013) Does C-reactive protein predict the long-term progression of interstitial lung disease and survival in patients with early systemic sclerosis? Arthr Care Res (Hoboken) 65:1375-1380. https://doi.org/10.1002/acr.21968

43. Åttingsberg E, Hoyer N, Wilcke T, Prior TS, Bendstrup E, Shaker $S$ (2019) Lactate dehydrogenase as a biomarker of advanced disease in idiopathic pulmonary fibrosis. In: Idiopathic interstitial pneumonias [Internet]. European Respiratory Society, p PA4701. http://erj.ersjournals.com/lookup/doi/10.1183/13993003.congr ess-2019.PA4701

44. Gharsalli H, Mlika M, Sahnoun I et al (2018) The utility of bronchoalveolar lavage in the evaluation of interstitial lung diseases: a clinicopathological perspective. Semin Diagn Pathol 35:280-287. https://doi.org/10.1053/j.semdp.2018.08.003

Publisher's Note Springer Nature remains neutral with regard to jurisdictional claims in published maps and institutional affiliations.

\section{Authors and Affiliations}

\section{David Lang ${ }^{1}$ (D) Kaveh Akbari ${ }^{2} \cdot$ Andreas Horner $^{1,4} \cdot$ Magdalena Hepp $^{1} \cdot$ Bernhard Kaiser $^{1} \cdot$ Herwig Pieringer $^{3} \cdot$ Bernd Lamprecht ${ }^{1}$}

Kaveh Akbari

radiologie@kepleruniklinikum.at

Andreas Horner

lunge@kepleruniklinikum.at

Herwig Pieringer

herwigpi@yahoo.com

1 Department of Pulmonology, Kepler University Hospital GmbH, Krankenhausstrasse 9, 4020 Linz, Austria
2 Central Radiology Institute, Kepler University Hospital GmbH, Krankenhausstrasse 9, 4020 Linz, Austria

3 Diakonissen Hospital Linz, Weissenwolfstrasse 15, 4020 Linz, Austria

4 Institute of General, Family and Preventive Medicine, Paracelsus Medical University, Strubergasse 21, 5020 Salzburg, Austria 